\title{
Survival, disabilities in activities of daily living, and physical and cognitive functioning among the oldest-old in China: a cohort study
}

\author{
Yi Zeng, Qiushi Feng, Therese Hesketh, Kaare Christensen, James W Vaupel
}

\section{Summary}

Background The oldest-old (those aged $\geq 80$ years) are the most rapidly growing age group globally, and are most in need of health care and assistance. We aimed to assess changes in mortality, disability in activities of daily living, and physical and cognitive functioning among oldest-old individuals between 1998 and 2008.

Methods We used data from the Chinese Longitudinal Healthy Longevity Study. Three pairs of cohorts aged 80-89 years, 90-99 years, and 100-105 years (in total, 19528 oldest-old participants) were examined; the two cohorts in each pair were born 10 years apart, with the same age at the time of the assessment in the 1998 and 2008 surveys. Four health outcomes were investigated: annual death rate, Activities of Daily Living (ADL), physical performance in three tests and cognitive function measured by Mini-Mental State Examination (MMSE). We used different tests and multivariate regression analyses to examine the cohort differences.

Findings Controlling for various confounding factors, we noted that annual mortality among oldest-old individuals was substantially reduced between $0.2 \%$ and $1.3 \%$ in 1998-2008 compared with individuals of the same age born 10 years previously, and that disability according to activities of daily living had significantly reduced annually between $0 \cdot 8 \%$ and $2 \cdot 8 \%$. However, cognitive impairment in the later cohorts increased annually between $0 \cdot 7 \%$ and $2 \cdot 2 \%$ and objective physical performance capacity (standing up from a chair, picking up a book from the floor, and turning around $360^{\circ}$ ) decreased anually between $0.4 \%$ and $3.8 \%$. We also noted that female mortality was substantially lower than male mortality among the oldest-old, but that women's functional capacities in activities of daily living, cognition, and physical performance were worse than their male counterparts.

Interpretation Advances in medications, lifestyle, and socioeconomics might compress activities of daily living disability, that is, benefits of success, but lifespan extension might expand disability of physical and cognitive functioning as more frail, elderly individuals survive with health problems, that is, costs of success.

Funding National Natural Science Foundation of China, National Institute on Aging/National Institutes of Health, United Nations Funds for Population Activities.

\section{Introduction}

Population aging is one of the major challenges facing most countries in the world, including China. The accompanied dramatic increase in numbers of the oldestold (individuals older than 80 years) is of particular concern, presenting a major challenge for health and social care systems, because the oldest-old often need daily assistance and medical care. ${ }^{1}$ Two contrasting scenarios of health trends in aging populations have been proposed. One view states that advances in medical technology, improvements in lifestyle, and socioeconomic development will postpone the onset of disability and chronic diseases among the elderly, so that morbidity will be compressed in old age. ${ }^{2-4}$ This concept is linked to the benefits of success-ie, that people are living longer (success) and in better health at older ages than they were previously (benefits). By contrast, in the alternative scenario, reduced mortality is hypothesised to result in an increased number of frail elderly people surviving with health problems, thus worsening the overall health of the elderly population. This concept is often referred to as expansion of morbidity, ${ }^{5,6}$ closely linked to that of costs of success, which specifically means that people's lifespans are lengthening (success) but with worse health at older ages than previously (costs). In reality, these two trends might coexist and interplay, ${ }^{7}$ and the concept of dynamic equilibrium has been introduced to help understand the association between morbidity and increasing life expectancy. ${ }^{8}$

Trends in the overall health status of the elderly population are generally positive in high-income societies. ' However, several reports support the opposite trend for some major health indicators. For example, findings from a Swedish study showed that the objective function tests of physical capacity, lung function, and cognition were significantly worse in 2002 compared with 1992 in individuals older than 77 years. ${ }^{10}$ Although dementia incidence has fallen in some European countries $^{11}$ and the USA, ${ }^{12}$ findings from nine large Japanese studies ${ }^{13}$ have suggested that prevalence of allcause dementia and Alzheimer's disease are increasing in Japan. Investigators building on the work of several 
For the Wan Fan database

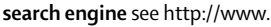
wanfangdata.com.cn/ For the Knowledge Network search engine see http://www. cnki.net/
Research in context

\section{Evidence before this study}

We used PubMed, Web of Science, and Google Scholar to search for publications before Aug 30, 2016 in English. The Chinese literature was identified through searches of the Wan Fan database search engine and the Knowledge Network search engine. We used the search terms "oldest-old", "old age", "mortality", "ADL", "cognitive function", "physical performance", and "cohort differences", etc. We also checked the reference lists of the related publications identified in the search. Existing scientific literature has provided empirical support for both compression of morbidity (including disability and chronic diseases) and expansion of morbidity as human longevity increases. However, no research so far has investigated the mixed effects of these two opposing trends in a single study with a large enough sample size of the oldest-old cohorts, except one study of Danish cohorts.

\section{Added value of this study}

Our findings support that, with increased longevity (success), there are co-existence and mixed effects of compression of disability in activities of daily living (benefits of success) and expansion of disability in physical and cognitive functioning (costs of success), which is in general consistent with findings from the Danish study that compared one pair of cohorts born 10 years apart aged 93 or 95 years in 1998 or 2010, with a total sample size of 5430 nonagenarians. However, we also noted important differences. The novelty of this study is that we compare three groups of Chinese cohorts born 10 years apart aged $80-89$ years, $90-99$ years, and $100-105$ years at the time of the surveys in 1998 or 2008 , with a total sample of 19528 oldest-old participants. To our knowledge, this study is the first investigation of this important issue based on the largest dataset of oldest-old cohorts in the world and from a low-income or middle-income country.

Implications of all the available evidence

The combination of declining mortality with worsening cognition and physical performance among the rapidly growing population of oldest-old individuals has clear policy implications for health systems and social care, not only in China but also globally. Many more state-subsidised public and private programmes and enterprises are urgently needed to provide services to meet the various needs of the rapidly growing elderly population, especially the oldest-old. Additionally, programmes to prevent chronic disease in elderly people through individualised health interventions need to be prioritised. studies (including two nationally representative surveys) reported opposing trends of improvement in disability measures, alongside an expansion of morbidity in chronic diseases and functional impairments, among Swedish oldest-old. ${ }^{10,14}$

Several studies have reported that the prevalence of disability according to activities of daily living among Chinese elderly people has decreased in the past two decades..$^{15,16}$ However, Wu and colleagues ${ }^{17}$ concluded that dementia prevalence among elderly individuals aged 70 years or older was generally increasing, on the basis of an evaluation of 70 prevalence studies of dementia in mainland China, Hong Kong, and Taiwan from 1980 to 2012. ${ }^{17}$ Similarly, Chan and colleagues ${ }^{18}$ reported that the prevalence of all forms of dementia at ages 65-69 years and 95-99 years in China in 2010 had increased by $44.4 \%$ and $43.7 \%$, respectively, compared with $1990 .{ }^{18}$

The existing scientific literature provides empirical support for both compression and expansion of morbidity, but little research so far has investigated the mixed effects of these two opposing trends in a single study with a sufficient sample size of the oldestold. The exception is a Danish study of a cohort born in 1905 and assessed at age 93 in 1998, compared with a later cohort born in 1915 and assessed at age 95 in 2010. ${ }^{19}$ This study provided some support for the mixed effects of both compression of morbidity and expansion of morbidity. However, whether these mixed effects also exist among the oldest-old in low-income or middle-income countries such as China is unclear. We aimed to address this research question by comparatively analysing cohorts of the oldest-old born in 1909-18 versus 1919-28 (aged 80-89 years in 1998 vs 2008), born in 1899-1908 versus 1909-18 (aged 90-99 years in 1998 vs 2008), and born in 1893-98 versus 1903-08 (aged 100-105 years in 1998 vs 2008). To our knowledge, this study is the first to assess this important issue in a lowincome or middle-income country, and uses the largest dataset of oldest-old cohorts in the world.

\section{Methods \\ Study design and participants}

This study draws on data from the oldest-old participants (ie, those aged 80-105 years) from the 1998 and 2008 waves of the Chinese Longitudinal Healthy Longevity Surveys (CLHLS). The CLHLS is a nationwide survey done in a randomly selected half of the counties and cities in 22 of the 31 provinces, covering about $85 \%$ of the total population of China. The CLHLS attempted to interview all centenarians who voluntarily agreed to participate in the study in the sampled counties and cities. The CLHLS also adopted a targeted random-sample design to ensure representativeness, through interviews with approximately equal numbers of male and female nonagenarians, octogenarians, and young-old (aged 65-79 years) living near to the centenarians (ie, in the same village or street, if available, or in the same sampled county or city). This design serves well our aim of 
investigating determinants of healthy longevity of different age and sex groups who live in the same social and natural environment. ${ }^{20}$

The Research Ethics Committees of Peking University and Duke University granted approval for the Protection of Human Subjects for the Chinese Longitudinal Healthy Longevity Survey, including collection of the data used for present study. The survey respondents gave informed consent before participation.

\section{Procedures}

The CLHLS was initially designed to facilitate international comparative analyses, and its questionnaire was translated from the instruments of the Danish longevity survey analysed by Christensen and colleagues. ${ }^{19}$ The instruments were adapted to the Chinese culture and socioeconomic context. A wide variety of international and domestic studies have confirmed that age reporting of the Han Chinese oldestold is in general reasonably accurate, due to the cultural tradition of memorising one's date of birth to determine dates of important life events such as engagement and marriage. .1,22 $^{2}$

The CLHLS 1998 and 2008 surveys used almost exactly the same ascertainment and assessment protocols. No proxy was used for objective questions such as assessment of cognitive function and physical performance. The survey was administered in the participants' homes by trained interviewers from the local centres for disease prevention and control for university students. More details about CLHLS, including sampling design, follow-up interviews with surviving participants and deceased participants' close family members, data quality, and the variables analysed are described in the appendix.

\section{Statistical analyses}

We divided individuals into oldest-old born in 1909-18 versus 1919-28 (aged 80-89 years in 1998 vs 2008), born in 1899-1908 versus 1909-18 (aged 90-99 years in 1998- 2008), and born in 1893-98 versus 1903-08 (aged 100-105 years in 1998 vs 2008).

We compared annual mortality, self-reported disability according to the activities of daily living scale, physical performance in three tests, and cognitive function measured by Mini Mental State Examination scores for men and women separately and for both sexes combined. We did standard statistical $\chi^{2}$ tests (one-sided) or $Z$ tests (two-sided) for categorical data, and $t$ tests (two-sided) for continuous data. We also did multivariate regression analyses to explore the changes in mortality, physical function, and cognitive function between the oldest-old cohorts born 10 years apart, adjusted for the covariates of age, rural or urban residence, marital status, and education, which are the major demographic and socioeconomic factors affecting the mortality and health of elderly people in China. We based the mortality analysis on parametric survival models with Weibull distribution, while the Weibull assumption was satisfied. All other analyses were based on logistic regression models or linear regression models. We used STATA version 13.1 for the statistical analyses.

\section{Role of funding source}

The study funders provided financial support for data collection and analysis, but had no role in the writing of the report, interpretation of the results, or submission for consideration of publication. The corresponding author had full access to all the data in the study and had final responsibility for the decision to submit for publication.

\section{Results}

We included 19528 individuals in our study, comprising 7288 octogenarians, 7234 nonagenarians, and 5006 centenarians, interviewed in 1998 and 2008 (in view of the very high mortality at advanced ages, only $2 \cdot 8 \%$ of the oldest-old participants were interviewed in both 1998 and 2008 surveys). The appendix presents the basic demographic characteristics of the cohorts. Tables 1-3 present the detailed results of cross-cohort changes in physical and cognitive function and death during followup for men, women, and both sexes combined. Figures 1, 2 and table 4 present the summary results.

Age-specific and sex-specific mortality among Chinese oldest-old aged $80-89$ years, $90-99$ years, and $100-105$ years were all reduced in the later cohorts compared with the cohorts born 10 years earlier (figure 1; tables 1-3). All of the nine sets of comparisons of age-specific mortality between the different cohorts of the oldest-old showed reductions of $-0 \cdot 2 \%$ to $-1 \cdot 3 \%$ in annual mortality during follow-up (table 4). Adjusted for covariates of age, sex, education, and rural or urban residence, the cross-cohort reduction in age-specific and sex-specific mortality was statistically significant in sex-combined centenarians $(\mathrm{p}=0.0032)$ and female centenarians $(\mathrm{p}=0.0163)$, and not significant in sex-combined octogenarians and nonagenarians, male or female octogenarians, male or female nonagenarians, or male centenarians (tables 1-3).

Disability as measured through activities of daily living of the Chinese oldest-old was significantly reduced in the later cohorts compared with the earlier cohorts (figure 1, tables 1-3). All of the nine sets of comparisons between different cohorts of the oldest-old showed substantial reductions in annual rates of disability, ranging from $-0 \cdot 8 \%$ to $-2 \cdot 8 \%$ (table 4 ). Adjusted for the covariates, the cross-cohort reductions in the mean score of activities of daily living disability were statistically significant $(\mathrm{p}<0.0001)$ for nonagenarians and centenarians (both sexes combined) and female nonagenarians and centenarians, significant (ranging from $\mathrm{p}=0.0023$ to $\mathrm{p}=0.0082$ ) in sex-combined octogenarians and male nonagenarians, significant (ranging from $\mathrm{p}=0 \cdot 0257$ to $\mathrm{p}=0 \cdot 0290$ ) in female and 


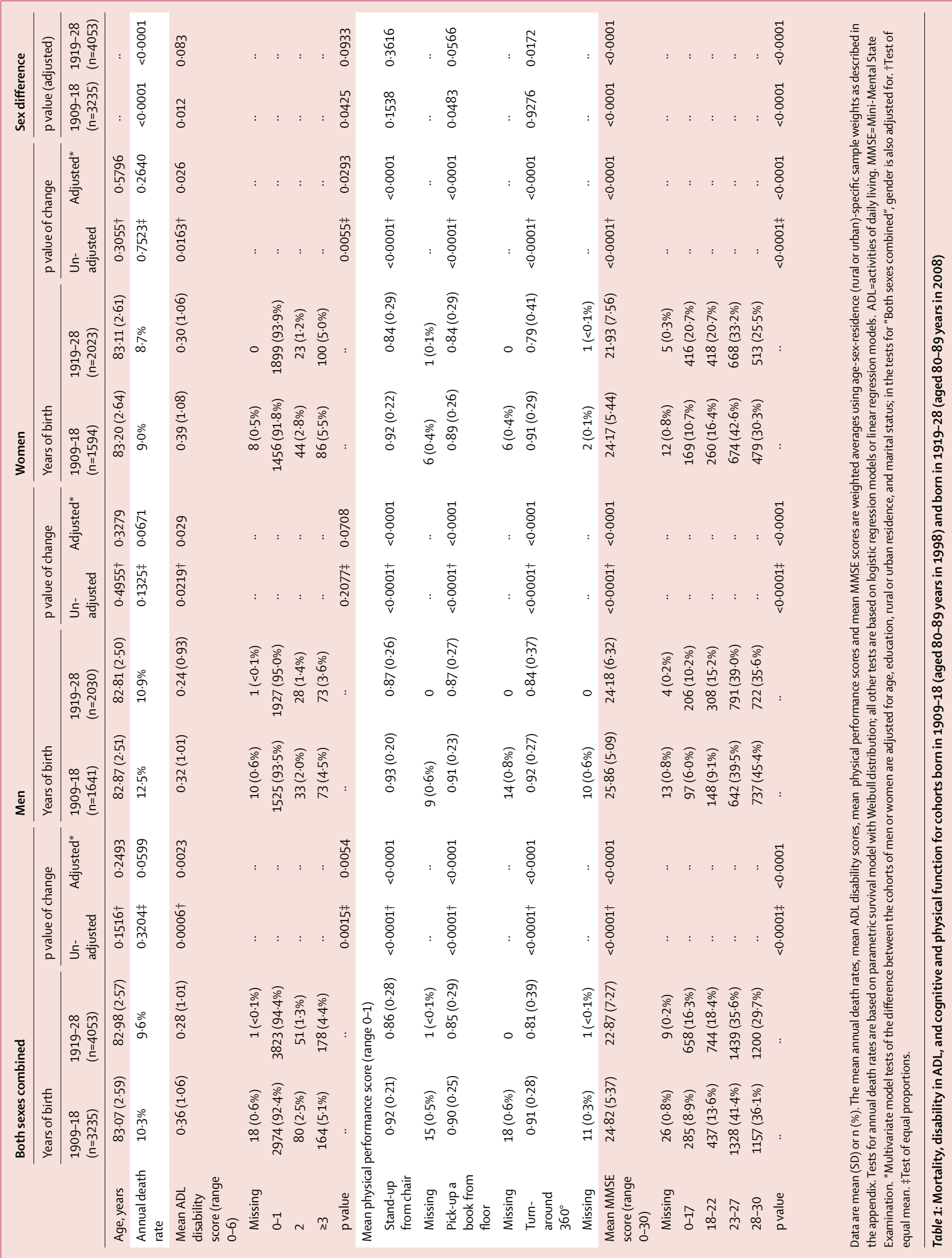




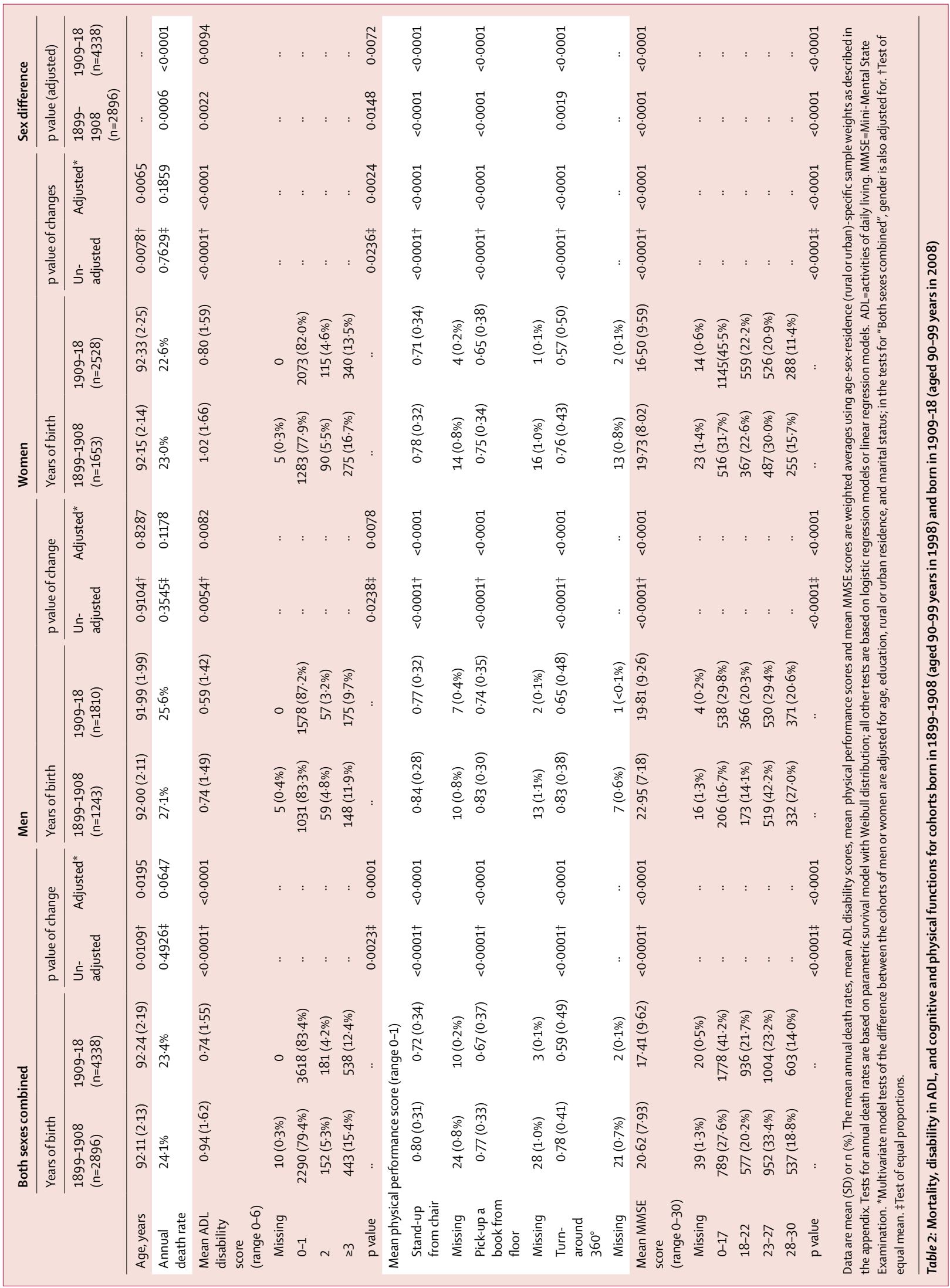




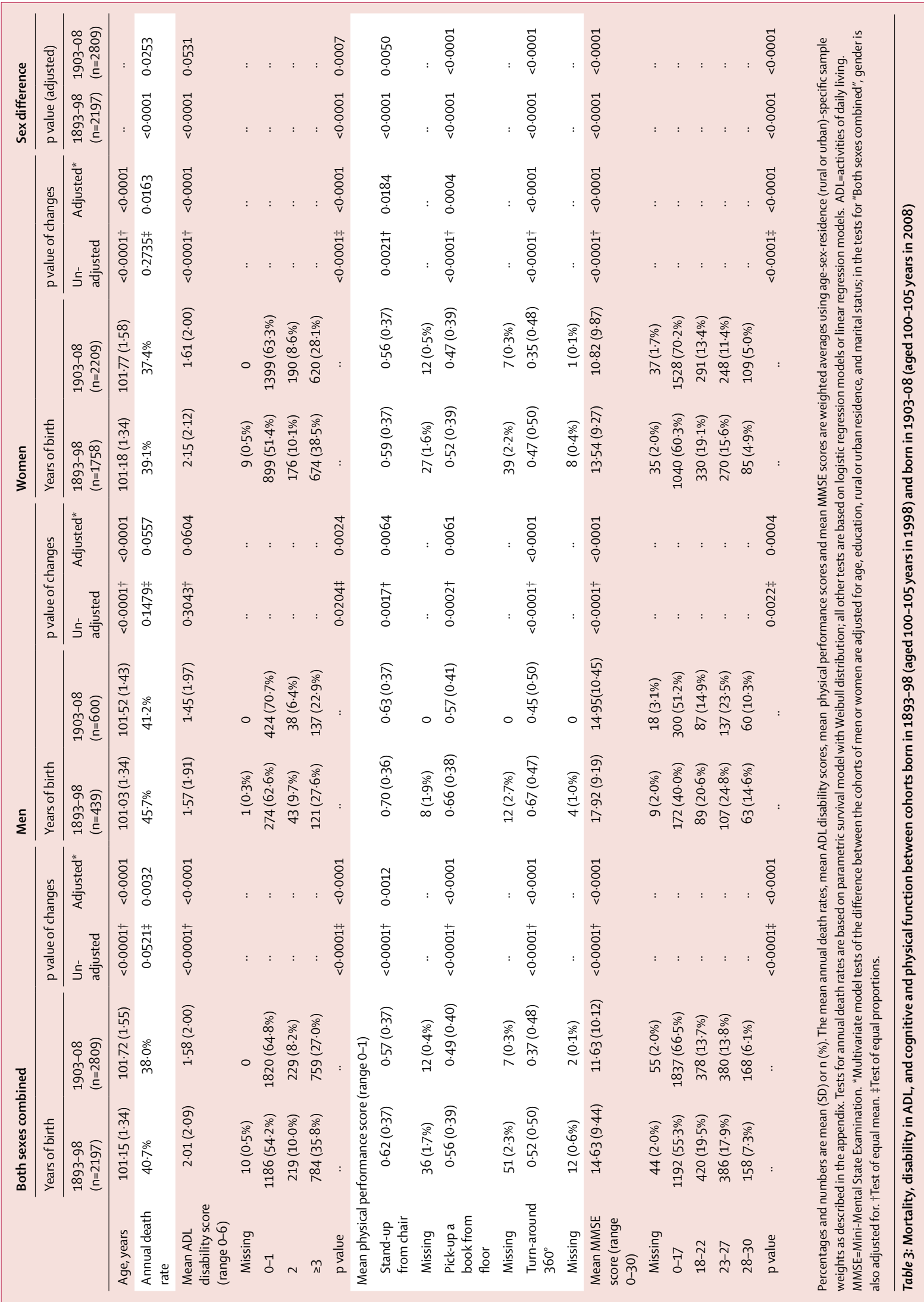


male octogenarians, and not significant in male centenarians $(\mathrm{p}=0 \cdot 0604$; tables $1-3)$.

The scores in objective physical performance tests (standing up from a chair, picking up a book from the floor, and turning-around $360^{\circ}$ among the Chinese oldestold were all significantly worsened in the later cohorts compared with the earlier cohorts (figure 2, tables 1-3). All of the 27 sets of comparisons of physical performance tests between different cohorts of the oldest-old showed substantial reductions in annual rates, from $-0 \cdot 4 \%$ to $-3 \cdot 8 \%$ (table 4 ). Adjusted for the covariates, the cross-cohort differences in objective physical performance were highly significant in octogenarians, nonagenarians, and centenarians for men, women, and both sexes combined $(\mathrm{p}<0.0001)$ in 22 comparisons, ranging from $\mathrm{p}=0 \cdot 0004$ to $\mathrm{p}=0 \cdot 0064$ in four comparisons, and $\mathrm{p}=0 \cdot 0184$ in one comparison; tables 1-3).

The cognitive function measured by the Mini-Mental State Examination test scores of the Chinese oldest-old was significantly worse in the later cohorts compared with the earlier cohorts (figure 2, tables 1-3). All of the nine sets of comparisons of cognitive function between different cohorts of the oldest-old showed significant reductions in annual rates, ranging from $-0 \cdot 7 \%$ to $-2 \cdot 2 \%$ (table 4 ). Adjusted for the covariates, the cross-cohort differences in cognitive functional scores were statistically significant $(\mathrm{p}<0 \cdot 0001)$ in all of the nine comparisons for octogenarians, nonagenarians, and centenarians, for both sexes and for men and women separately (tables 1-3).

Tables 1-3 show male-female comparisons of the six pairs of oldest-old cohorts aged 80-89 years, 90-99 years, and 100-105 years in 1998 and 2008; men had substantially and consistently higher age-specific mortality than did women, but substantially better health status in terms of ADL disability, physical performance test scores, and cognitive function. The sex differences in the 48 male-female comparisons were statistically significant (mostly $\mathrm{p}<0 \cdot 001$ ), except six non-significant comparisons in octogenarians and one non-significant comparison in centenarians.

Self-reported life satisfaction and self-reported good health significantly declined among the later oldestold cohorts compared with the earlier oldest-old cohorts (appendix; $\mathrm{p}<0.0001$ ), except self-reported health in centenarians. Of note, the period cross-sectional comparisons showed that average self-reported life satisfaction and health slightly increased or remained almost the same from ages 80-89 years to 90-99 years and 100 years or older in both the 1998 and 2008 surveys (appendix), whereas scores for disability as measured by activities of daily living, cognitive function, and physical performance were all largely increased with increased age (appendix).

\section{Discussion}

In this cohort study we compared three groups of Chinese individuals born 10 years apart, aged $80-89$ years,
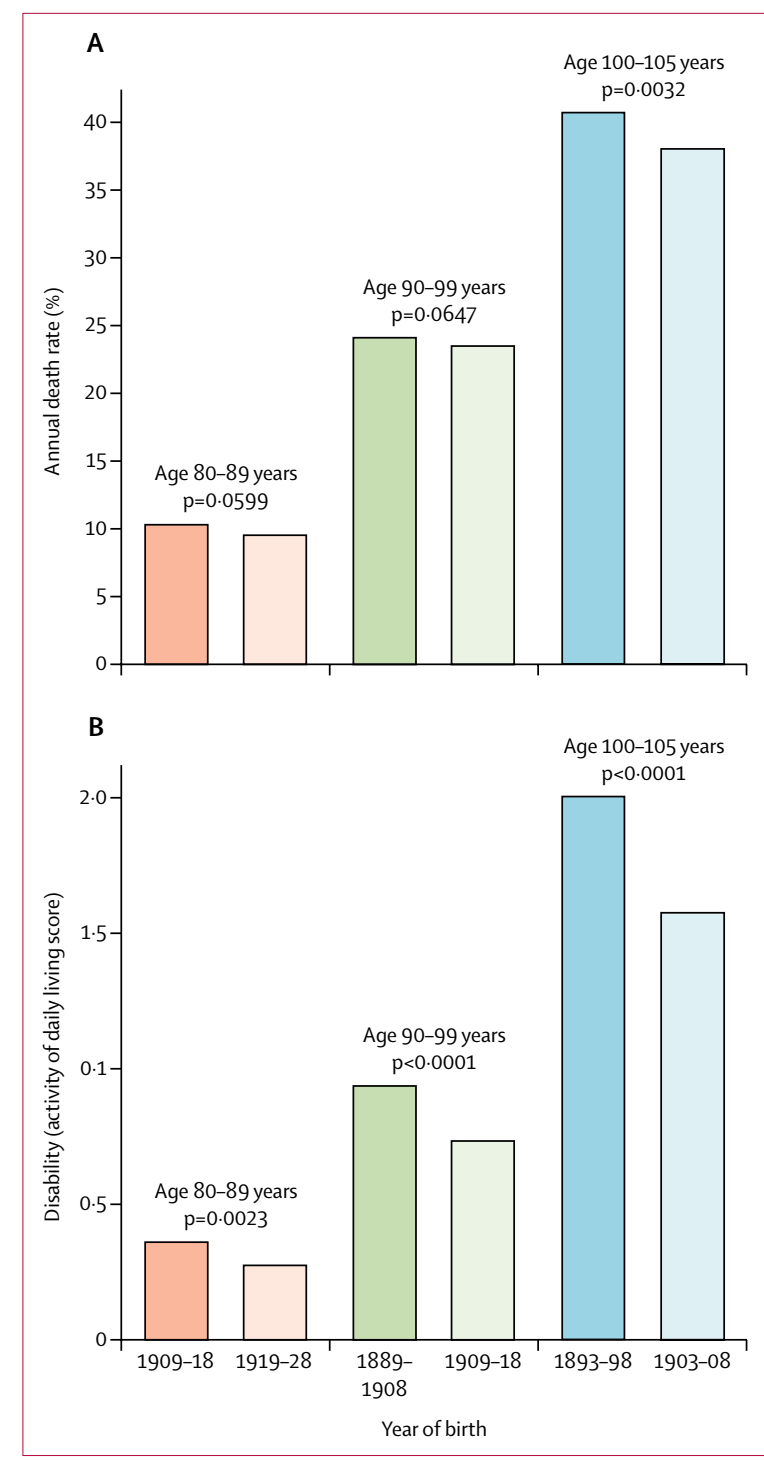

Figure 1: Annual death rates and disability in activities of daily living compared within three pairs of cohorts

Figure shows data for both sexes combined.

90-99 years, or 100-105 years at the time of surveys done in 1998 or 2008 . To our knowledge, this study is the first to assess this important issue in a low-income or middleincome country, and uses the largest dataset of oldest-old cohorts in the world. Our findings are generally consistent with those from the Danish study that compared a pair of cohorts born 10 years apart and aged 93 or 95 in 1998 or 2010. ${ }^{19}$ However, we also noted important differences.

Findings from both our Chinese study and the Danish study showed that mortality and disability (as defined by activities of daily living) among the later cohorts of the oldest-old were substantially reduced compared with the cohorts born 10 years earlier. However, the objective scores for physical performance (standing up from a 

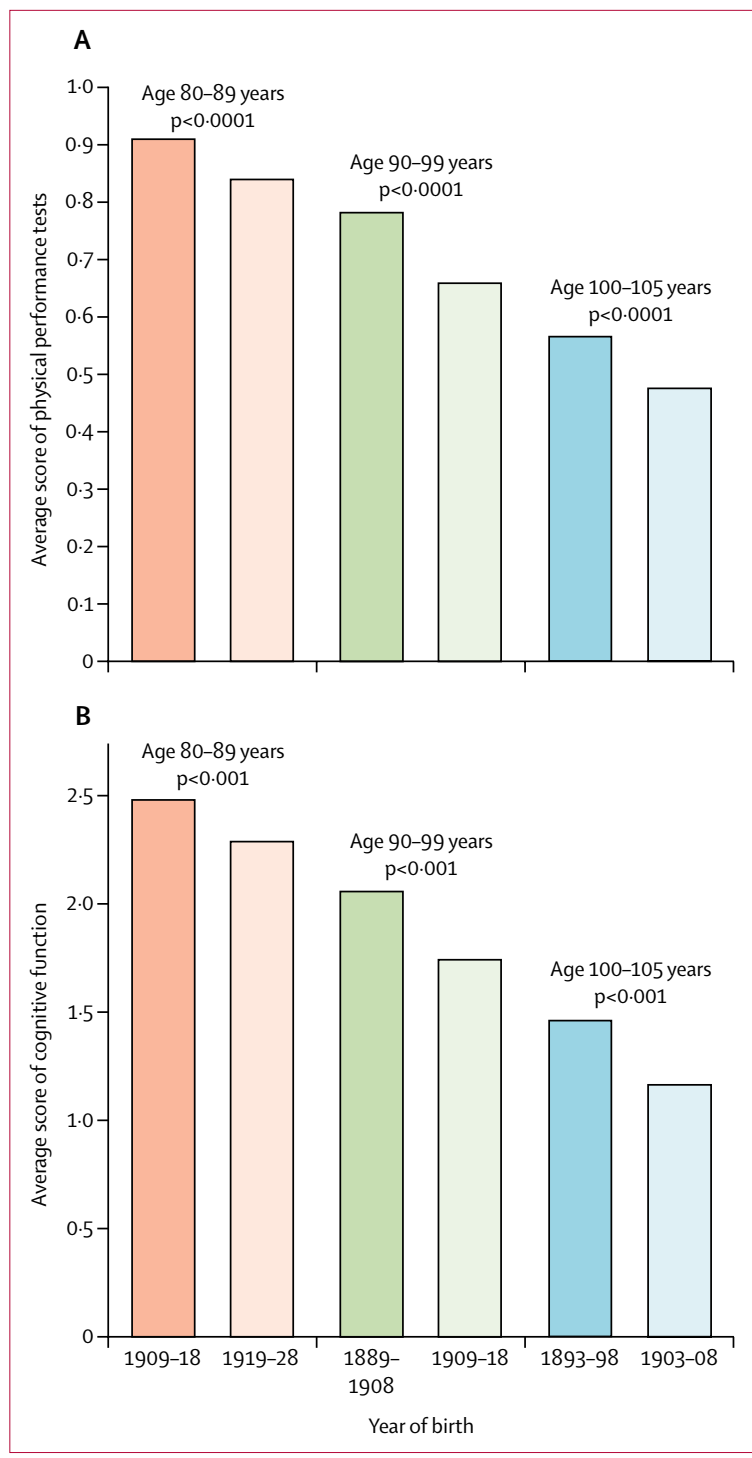

Figure 2: Objective physical performance tests and cognitive function compared within the three cohorts

Figure shows data for both sexes combined.

chair, picking up a book from the floor, and turning around $360^{\circ}$ ) for the Chinese oldest-old were all significantly worse in the later cohorts compared with the earlier cohorts. This observation is mostly consistent with the general pattern in the Danish study. Compared with the earlier cohort, the Danish later cohort had substantially worse ability to stand up from a chair or walk for $3 \mathrm{~m}$ among women and for both sexes combined. ${ }^{19}$

Both this study and the Danish study showed apparently contradictory findings with respect to survival and self-reported activities of daily living versus objective physical performance tests. We believe that two underlying factors might help to understand this effect. The first is the mixed effects of the two opposing processes of compression of morbidity (ie, benefits of success) and expansion of morbidity (ie, costs of success). On one hand, the later cohorts might benefit from progress in effective disease treatment, healthier lifestyles, declining disability effects of some major chronic diseases (eg, stroke and cardiometabolic disease), ${ }^{15}$ and improved standards of living due to rapid socioeconomic development in China. These benefits of success imply that the later cohorts of oldest-old individuals show reduced mortality due to postponement of senescence, and have reached older ages with improved health and functional capacity in daily living. ${ }^{4}$ On the other hand, as compared with the earlier cohort, the later cohort includes more members who have survived life-threatening conditions (because of improvements in medical care and increased longevity), but they might be in relatively poor health, implying that the saving of lives might reduce overall physical functional capacity and health. ${ }^{19}$ We propose to use the term "costs of success" to describe this effect.

The second underlying factor is associated with different types of disability measurements. Disability as measured by self-reported activities of daily living depends not only on health status, but also on facilities to assist such activities (eg, transferring, using the toilet, and bathing). The substantial improvement of activities of daily living among the Chinese oldest-old could be partly due to the rapid changes in living standards and availability of facilities for daily life during the past few decades in China. For example, average annual disposable income among urban and rural households in 2008 was 3.0 times higher (urban) and 2.2 times higher (rural) than in $1998 .{ }^{23}$ Such rapid improvements in living standards that provide better facilities for daily life could help to explain the significant decreases in disability associated with activities of daily living. However, the objective tests of physical performance do not depend on facilities. Furthermore, self-reported activities of daily living are subject to substantially higher measurement errors compared with objectively-tested cognitive function and physical performance. ${ }^{24}$ Thus, disability scores based on self-reported activities of daily living might not be an accurate indicator of physical health status, although it can be used as a good measurement of assistance needs in daily living activities. The objective tests of physical performance have added predictive value beyond the self-reported measures of disability in evaluation of actual health status changes and decision making about health interventions. ${ }^{25}$

Cognitive function among the Chinese oldest-old was substantially and significantly worse in the later oldestold cohorts compared with the earlier cohorts, consistent with the trends reported in the other studies in mainland China, Hong Kong, and Taiwan. ${ }^{17,18}$ However, the Danish 1915 cohort scored significantly better for cognitive function (as assessed by the Mini-Mental State Examination) than did the 1905 cohort. $^{19}$ We believe the explanation for this disparity lies in cross-cohort 
differences in education. For the two sexes combined, the weighted average education levels of the three Chinese later cohorts born in 1903-08, 1909-18, or 1919-28 were significantly lower than that of the three corresponding cohorts born 10 years earlier, adjusted for age, sex, rural or urban residence, and marital status. The weighted average proportion of frequently going to bed hungry as a child (retrospectively self-reported) among the later cohorts was $30.5 \%$ higher than in the earlier cohorts. Such cross-cohort differences in educational attainment and childhood conditions probably resulted from the increase in domestic wars during the periods when the later cohorts were children, compared with the earlier cohorts. This finding implies that the poorer education, childhood conditions, and subsequent adult socioeconomic status experienced by the later cohorts contributed to their lower cognitive function score, as shown in other studies using the CLHLS data. ${ }^{26}$ This effect was in addition to the costs of success effects, which resulted in some frail elderly individuals being saved from dying but surviving with poor cognitive function. However, the average education level in the Danish 1915 cohort was significantly better than in the 1905 cohort $(\mathrm{p}=0 \cdot 006)$. Because higher education level is strongly associated with improved cognitive function in old age, ${ }^{27}$ the positive effects of increased education level in the Danish later cohort might surpass the negative effects of the costs of success on cognitive function.

We observed that the magnitude of the difference in changes of physical and cognitive functions between the later and earlier oldest-old cohorts was substantially larger in our Chinese study than in the Danish study. For example, the difference in annual rates of changes in disability of activities of daily living between the later and earlier cohorts among Chinese nonagenarians was $2.3 \%$, by contrast with the $1.1 \%$ reported for the Danish nonagenarian cohorts. Such differences in magnitude of changes are understandable, because China is still undergoing rapid health transition and socioeconomic development, whereas Denmark passed this stage a few decades ago.

Understandably, self-reported life satisfaction and good health substantially fell among the Chinese oldest-old cohorts interviewed in 2008 compared with the oldest-old cohorts interviewed in 1998 (appendix), because the later cohorts had significantly worse scores for physical performance and cognitive function and their expectancy for good life and health was higher with increased living standards. By contrast with the Chinese results, the opposite trend was observed in the Danish cohorts where the fraction of individuals with excellent self-reported health increased substantially (unpublished data).

The period cross-sectional comparisons among difference age groups interviewed in the same year (2008) showed that the scores measuring capacities of self-reported activities of daily living, objective physical performance, and cognitive function decreased largely

\begin{tabular}{|c|c|c|c|c|c|c|c|c|c|c|c|}
\hline 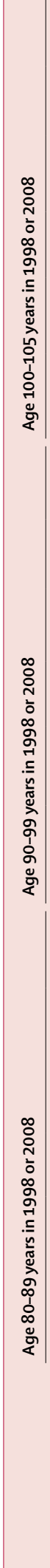 & 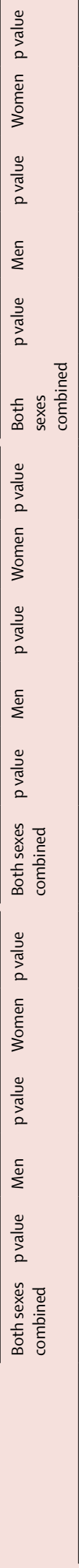 & 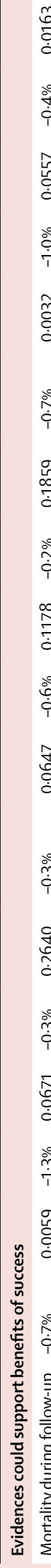 & 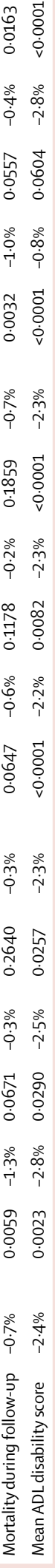 & 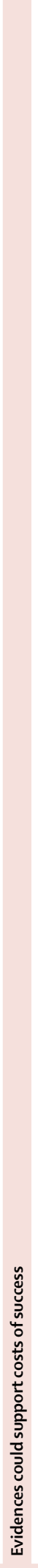 & 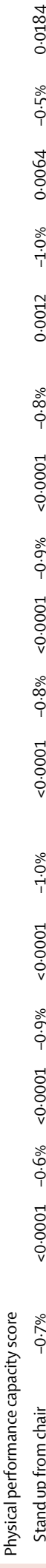 & 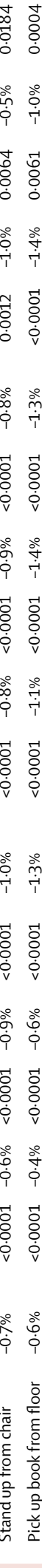 & 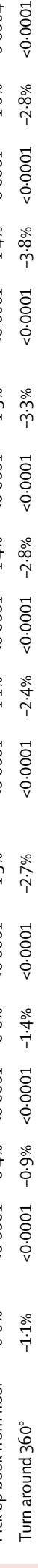 & $\begin{array}{l}\overrightarrow{8} \\
\dot{8} \\
\dot{0}\end{array}$ & & 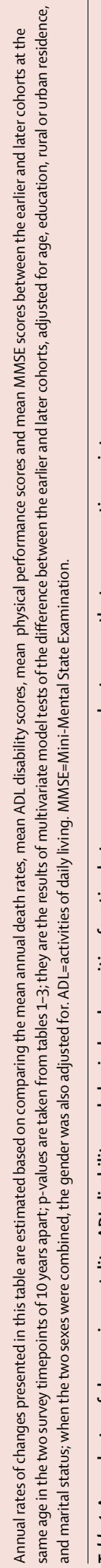 & 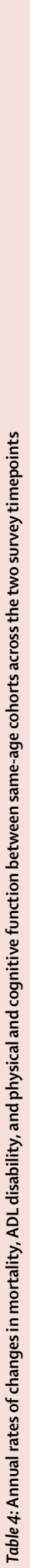 \\
\hline
\end{tabular}


from ages $80-89$ years to 100 years or older, but the proportion of the oldest-old individuals reporting satisfaction and good health slightly increased or remained almost unchanged across these ages (appendix). This finding is consistent with research about optimism and survival in the Danish 1905 cohort, ${ }^{28}$ and three Danish population-based surveys (including 11307 participants aged $\geq 45$ years, of whom 2411 were 90 years or older), which demonstrated that decline in activities of daily living and cognitive function did not necessarily affect happiness at older ages..$^{29}$ Our findings in the Chinese setting and the Danish findings might suggest that being more positive in one's outlook on life (ie, optimism and happiness) could increase the chance of longevity.

Our study has important limitations and further research is needed. For example, additional in-depth studies are warranted to develop a deeper understanding of the mechanisms and causalities of how and why mortality and activities ofdaily living disability risk significantly declined due to benefits of success, whereas the objective tests of physical performance and cognitive function were significantly reduced due to the effects of costs of success. We did not investigate trends in the prevalence of clinically diagnosed chronic diseases (an important aspect of morbidity) between the earlier and later cohorts, and we did not make comparisons for the representative samples of young-old cohorts born 10 years apart because of insufficient data (appendix). Further studies need to extend our analysis to cover all elderly age groups and include chronic diseases to fully understand the process of healthy aging in a lifecourse perspective, with a sufficiently large sample size of both oldest-old and young-old cohorts. In-depth research into the trends between various health outcome indicators might provide solid evidence for health intervention programmes aimed at strengthening the positive effects of the benefits of success and reducing the negative effects of the costs of success. This research would fundamentally contribute to the sustainable development of human societies in the face of worldwide rapid population aging with increased longevity.

Populations in China and many other countries in the world are aging rapidly. The oldest-old will increase much faster than any other age groups. The findings of the present study and other research ${ }^{10,13,14,17-19,30}$ provide a clear warning message to societies with aging populations, namely that although individuals enjoy increasing longevity and improvement in some health indicators (benefits of success), other major health indicators can deteriorate (costs of success). This effect poses enormous challenges for health systems, social care, and families, not only in China but also globally, especially in other lowincome and middle-income countries. The situation calls for policy actions. Thus, we believe that, to fully harvest the benefits and reduce the costs of the success from increasing lifespans, it is crucially important to develop many more state-subsidised public and private programmes and enterprises to provide services to meet the various needs of the growing elderly populations of both the oldest-old and young-old in China and worldwide. These measures should include long-term and acute daily care and mobility aids for people with disabilities, working opportunities for those elderly individuals who are still active, service and individualised intervention programmes for social and leisure activities, continued learning, opportunities for tourism, psychological counselling, and remarriage bridge-building.

\section{Contributors}

YZ designed the study and drafted the paper. QF performed the statistical data analysis and worked with YZ to draft the paper. All authors discussed and contributed to the theoretical framework, interpretation of the results, and revised and gave final approval of this manuscript.

\section{Declaration of interests}

We declare no competing interests.

\section{Acknowledgments}

We thank Xiaomin Shi, Zhaoxue Yin, Jieshi Luo, and Yuebin Lv (China Center for Diseases Control and Prevention), Yuzhi Liu, Chunyuan Zhang, Yun Zhou, and Zhenzhen Zheng (Peking University), Zhenyu Xiao, Liqun Tao, Qin Xu, and Ye Yuan (Chinese Center for Aging Science Research), and Jie Zhan (China Social Science Academy) for their contributions to the Chinese Longitudinal Healthy Longevity Survey (CLHLS), and we thank all interviewees and their families for their voluntary participation in the CLHLS study. We are very grateful for comments provided by Danan $\mathrm{Gu}$ and research assistance provided by Huashuai Chen and Muqi Guo. This study was funded by the National Natural Science Foundation of China (71110107025, 71233001, 71490732), National Institute on Aging/National Institutes of Health

(R01AG023627-10; 2P01AG031719), and United Nations Funds for Population Activities.

References

1 Zeng Y, George LK. Population ageing and old-age insurance in China In: Dannefer D, Phillipson C, eds. The SAGE handbook of social gerontology. London: SAGE Publications, 2010: 420-30.

2 Fries JF. Aging, natural death, and the compression of morbidity. New Engl J Med 1980; 303: 130-35.

3 Christensen K, Doblhammer G, Rau R, Vaupel JW. Ageing populations: the challenges ahead. Lancet 2009; 374: 1196-208.

4 Vaupel JW. Biodemography of human ageing. Nature 2010; 464: 536-42.

5 Gruenberg EM. The failures of success. Milbank Q 1977; 55: 3-24.

6 Waidmann T, Bound J, Schoenbaum M. The illusion of failure: Trends in the self-reported health of the US elderly. Milbank Q 1995; 73: 253-87.

7 Manton KG. Changing concepts of morbidity and mortality in the elderly population. Milbank Q 1982; 60: 183-244.

8 Robine JM, Michel JP. Looking forward to a general theory on population aging. J Gerontol A-Biol Sci 2004; 59: 590-97.

9 Freedman VA, Martin LG, Schoeni RF. Recent trends in disability and functioning among older adults in the United States: a systematic review. JAMA 2002; 288: 3137-46.

10 Parker MG, Ahacic K, Thorslund M. Health changes among Swedish oldest old: prevalence rates from 1992 and 2002 show increasing health problems. J Gerontol A-Med Sci 2005; 60: 1351-55.

11 Matthews FE, Stephan BCM, Robinson L, et al. A two decade dementia incidence comparison from the Cognitive Function and Ageing Studies I and II. Nat Commun 2016; 7: 11398.

12 Satizabal CL, Beiser AS, Chouraki V, et al. Incidence of dementia over three decades in the Framingham Heart Study. N Engl J Med 2016; 374: 523-32.

13 Dodge HH, Buracchio TJ, Gwenith GF, et al. Trends in the prevalence of dementia in Japan. Int J Alzheimers Dis 2012 2012: 956354. 
14 Parker MG, Thorslund M. Health trends in the elderly population: getting better and getting worse. Gerontologist 2007; 47: 150-58.

15 Liang Y, Song A, Du S, Guralnik JM, Qiu C. Trends in disability in activities of daily living among Chinese older adults, 1997-2006: the China Health and Nutrition Survey. J Gerontol A-Med Sci 2015; 70: 739-45.

16 Martin L, Feng Q, Scheoni B, Zeng Y. Trends in function and activity limitations among Chinese oldest-old, 1998 to 2008. Popul Dev Rev 2014; 40: 475-95.

17 Wu YT, Lee H, Norton S, et al. Period, birth cohort and prevalence of dementia in mainland China, Hong Kong and Taiwan: a meta-analysis. Int J Geriatr Psychiatry 2014; 29: 1212-20.

18 Chan KY, Wang W, Wu JJ, et al. Epidemiology of Alzheimer's disease and other forms of dementia in China, 1990-2010: a systematic review and analysis. Lancet 2013; 381: 2016-23.

19 Christensen K, Thinggaard M, Oksuzyan A, et al. Physical and cognitive functioning of people older than 90 years: a comparison of two Danish cohorts born 10 years apart. Lancet 2013; 382: $1507-13$

20 Zeng Y. Towards deeper research and better policy for healthy aging - using the unique data of Chinese Longitudinal Healthy Longevity Survey. China Economic J 2012; 5: 131-49.

21 Coale AJ, Li S. The effect of age misreporting in China on the calculation of mortality rates at very high ages. Demography 1991; 28: 293-301.

22 Wang Z, Zeng Y, Jeune B, Vaupel JW. Age validation of Han Chinese centenarians. GENUS 1998; 54: 123-41.
23 National Statistical Bureau of China. http://www.stats.gov.cn/tjsj/ tjgb/ndtjgb/qgndtjgb/200203/t20020331_30012.html (accessed May 25, 2016).

24 Theou O, O'Connell MD, King-Kallimanis BL, et al. Measuring frailty using self-report and test-based health measures. Age Ageing 2015; 44: 471-77.

25 Feng, Q, Hoenig HM, Gu D, Zeng Y, Purser JL. Impact of new disability subtypes on three-year mortality in Chinese older adults. J Am Geriatr Soc 2010; 58: 1952-58.

26 Zeng Y, Gu D, Land KC. The association of childhood socioeconomic conditions with healthy longevity at the oldest-old ages in China. Demography 2007; 44: 497-518.

27 Huang W, Zhou Y. Effects of education on cognition at older ages: evidence from China's great famine. Soc Sci Med 2013; 98: 54-62.

28 Engberg H, Jeune B, Andersen-Ranberg K, et al. Optimism and survival: does an optimistic outlook predict better survival at advanced ages? A twelve-year follow-up of Danish nonagenarians. Aging Clin Exp Res 2013; 25: 517-25.

29 Vestergaard S, Thinggaard M, Jeune B, et al. Physical and mental decline and yet rather happy? A study of Danes aged 45 and older. Aging Ment Health 2015; 19: 400-08.

30 Verbrugge LM, Brown DC, Zajacova A. Disability rises gradually for a cohort of older Americans. J Gerontol B Psychol Sci Soc Sci 2016 published online March 11. http://dx.doi.org/doi:10.1093/geronb/ gbw002. 